\title{
Inbreeding and population structure in two pairs of cryptic fig wasp species
}

\author{
DRUDE MOLBO, ${ }^{*}+$ CARLOS A. MACHADO§,EDWARD ALLEN HERRE† and LAURENT KELLER* \\ *Institute of Ecology, University of Lausanne, 1015 Lausanne, Switzerland; +Smithsonian Tropical Research Institute, Apartado 2072, \\ Balboa, Republic of Panama; $¥$ I.C.A.P.B., University of Edinburgh, King's buildings, Edinburgh EH93JT, Scotland, UK, §Department \\ Ecology and Evolutionary Biology, University of Arizona, Tucson, AZ 85721, USA
}

\begin{abstract}
We used recently developed microsatellites to directly estimate inbreeding levels in two pairs of coexisting cryptic fig wasp species ('Pegoscapus hoffmeyeri sp. A and sp. B', 'P. gemellus sp. $A$ and sp. $B$ '). Previous tests of Hamilton's local mate competition (LMC) theory in fig wasps have used the number of dead foundresses in a fig fruit to indirectly estimate the relative contribution of each to the common brood and thereby the level of local mate competition. Further, the population level of inbreeding has been indirectly estimated using the distribution of foundress numbers across broods. Our direct genetic estimates confirmed previous assumptions that the species characterized by lower foundress numbers showed higher relative levels of inbreeding. However, there were quantitative differences between the observed level of inbreeding and the expectation based on the distribution of foundress numbers in both pollinator species associated with Ficus obtusifolia. Here, genotype compositions of broods revealed that only $23 \%$ of fruits with multiple foundresses actually contained brood from more than one foundress, thus explaining at least part of the underestimate of actual sibmating. Within the four wasp species there was no evidence for genetic differentiation among the wasp populations sampled from different trees across $20 \mathrm{~km}$ and from different points in time. Further, no genotypic disequilibrium was detected within any of the species. Although F1 hybrids were observed between the two species pollinating F. obtusifolia, there was no evidence of genetic introgression. Finally, we found that $11 \%$ of the sons of allospecifically mated mothers were diploid hybrids suggesting a break down of the sex determination system in hybrids.

Keywords: Agaonidae, breeding structure, diploid male, $F_{\mathrm{ST}}$, hybridization, Local mate competition, Pegoscapus.
\end{abstract}

Received 14 September 2003; revision received 23 January 2004; accepted 23 January 2004

\section{Introduction}

Models of optimal sex allocation are among the very few evolutionary models that provide easily testable quantitative predictions, and they have proved successful at predicting sex allocation in a wide range of taxa (Fisher 1958; Hamilton 1967; Trivers \& Willard 1973; Trivers \& Hare 1976; Charnov 1982). This has made them textbook examples of the predictive power of evolutionary theory and much conceptual and empirical work is based on them

Correspondence: Drude Molbo. Present address: Smithsonian Tropical Research Institute, Naos, Apartado 2072, Balboa, Republic of Panama. Fax: (+ 507) 212 8791; E-mail: drudemolbo@hotmail.com, molbod@naos.si.edu
(Boomsma \& Grafen 1991; Hardy (ed.); Seger \& Eckhart 1996; West et al. 2000; 2002, Komdeur \& Pen 2002), Hamilton's local mate competition theory (LMC) is among the most notable of these models (Hamilton 1967; Werren 1983; Frank 1985; Herre 1985, 1987; West \& Herre 1998; Herre et al. 2001). LMC predicts the optimal sex ratio that a foundress (reproductive female) should lay on a patch of resources, when the offspring reared on that patch are restricted to mate among themselves. In the extreme case where she is alone on a patch, a foundress should produce only enough sons to fertilize all her daughters. However, the value of sons increases as the number of foundresses goes up because of the increased competition between unrelated males (Hamilton 1967). Consequently, the optimal sex ratio becomes less female biased with higher foundress numbers. 
In haplo-diploid species, optimal sex ratio further depends on the level of inbreeding. This is because inbreeding increases the relatedness asymmetry between a mother and her offspring of either sex. The haploid males develop from unfertilized eggs, so they carry only maternal genes and relatedness between mothers and sons does not change. Females, however, develop from fertilized eggs and with increasing inbreeding mothers are on average more related to their mates and thereby also more related to their diploid daughters. This increases the relative value of daughters compared to sons, and the optimal sex ratio is therefore more female-biased with higher levels of inbreeding (Hamilton 1979; Frank 1985; Herre 1985). The optimal sex ratio a foundress should lay is given by:

$r^{*}=\frac{1}{2}\left(\frac{1+F}{1+2 F}\right)\left(\frac{n-1}{n}\right)$

where $n$ is the foundress number and $F$ is the inbreeding coefficient (Hamilton 1979). The sex ratio is defined as the proportion of reproductive investment that is allocated to males. In fig wasps where there is no evidence of different resource allocation to the two sexes, this can be approximated by the proportion of males [males/(males + females)] (Frank 1985).

Fig wasps (Agaonidae, Chalcidoidea) have frequently been used for testing local mate competition because foundress numbers can be counted directly. Fig wasps are the sole pollinators of fig trees (Ficus spp.; Moraceae). One or a few foundresses enter a fig inflorescence (fruit) through a narrow opening. Once inside, they pollinate and lay their eggs in some of the flowers. In many species, foundresses die inside the inflorescences and hence can be counted at the time of offspring emergence. The offspring developing in a fruit is referred to as brood. After several weeks of development, the wingless males emerge first and mate with the females while these are still inside their galled flowers (personal observation, Frank 1984). As the females emerge and collect pollen, the males chew an exit hole through the wall of the fruit, which allows females to leave and search for new receptive inflorescences (Frank 1984).

The many studies of fig wasp sex ratios generally show qualitative agreement with both basic predictions of LMC theory. Within a species, as foundress number increases, sex ratios become less female biased (Frank 1985; Herre 1985, 1987; Herre et al. 1997, 2001; Kinoshita et al. 1998) Among species, as inbreeding estimates increase, sex ratios tend to become more female biased (Herre 1985, 1987; Herre et al. 1997, 2001). However, brood sex ratios were generally found to be more female biased than expected (Hamilton 1979; Frank 1985; Herre 1985, 1987; Herre et al. 1997, 2001; Kinoshita et al. 1998; Kathuria et al. 1999; see also Greeff 2002), raising the question of whether limitations in the theory or in the adaptive capacities of the wasps were responsible. Several authors have noted that both LMC and inbreeding have been estimated indirectly from the number of dead foundresses found in the fig fruits. These indirect estimates involve several assumptions, the violation of which weakens the confidence in tests of LMC using fig wasps. Explicitly, all dead foundresses in a fruit are usually assumed to have contributed equal numbers of offspring and sex ratios. Further, the offspring of foundresses should mate randomly within a fruit. This implicitly assumes that there is a single species of wasp pollinating each species of Ficus. Although there is reason to believe that the predictions of these models are fairly robust to violation of the underlying assumptions (Werren 1980; Frank 1985; Stubblefield \& Seger 1990; Greeff \& Compton 1996; Greeff 1997), if any of these assumptions are not met, then the power of the test is compromised. Thus, despite the general qualitative fit with theory, the previous indirect estimates of breeding structure need to be revised to test LMC and the precision of adaptation properly.

The aim of this study was to use recently developed microsatellites (Molbo et al. 2002) to characterize the levels of LMC and inbreeding independent of foundress numbers in the fig wasps (Pegoscapus spp.) pollinating two fig species. While conducting the genetic analyses we found that, contrary to the prevailing view, there were more than one species of wasp pollinating each of these two species of fig (Molbo et al. 2002; Molbo et al. 2003). In addition to the implications for earlier LMC tests, this introduces questions of genetic differentiation between populations and species and introgression between sympatric wasp species. Hence, we determined the rate of interspecific hybridization and the genetic population structure of each species.

\section{Materials and Methods}

\section{Collection}

We collected fig wasps from 1997 through 2000 from the two Neotropical hemi-epiphytic fig species, Ficus obtusifolia and F. popenoei (subgenus Urostigma; section Americana). All collections were made within $20 \mathrm{~km}$ of Barro Colorado Island, the Republic of Panama. Just prior to ripening but before wasp emergence, fig fruits were collected and brought back to the laboratory. The fruits were cut into halves and placed in sealed Petri dishes and the dead foundresses were counted. The offspring were allowed to emerge and then sexed, counted and stored in $95 \%$ alcohol pending DNA extraction.

Until proper names are given to the newly discovered species (Molbo et al. 2002; Molbo et al. 2003), we use the name of the species described by Wiebes (1995) followed by sp. A or sp. B. Ficus obtusifolia is pollinated by 'Pegoscapus hoffmeyeri spp. $A$ and $B$ ', and F. popenoei by 'P. gemellus spp. $A$ and $B$ '. We call these species 'cryptic', not in reference 
Table 1 Results of the population genetic analyses of four species of Pegoscapus wasps. $H_{\mathrm{T}}$ measures expected heterozygosity. $F_{\mathrm{IT}}, F_{\mathrm{ST}}$ and $F_{\mathrm{IS}}$ are given as means $\pm 95 \%$ confidence intervals after jackknifing over crops. In $P$. hoffmeyeri sp. $A$, where 5 loci were analysed, we also performed jackknifing over loci

\begin{tabular}{|c|c|c|c|c|c|c|c|c|}
\hline Locus & $\mathrm{N}^{\circ}$ of Alleles & Individuals & Crops & $N$ per crop & $H_{\mathrm{T}}$ & $F_{\text {IT }}$ & $F_{\mathrm{ST}}$ & $F_{\mathrm{IS}}$ \\
\hline \multicolumn{9}{|c|}{ P. hoffmeyeri sp. A } \\
\hline $\operatorname{Pe} 9$ & 18 & 339 & 14 & $10-44$ & 0.844 & $0.851 \pm 0.048$ & $0.009 \pm 0.011$ & $0.850 \pm 0.047$ \\
\hline Pe 28 & 4 & 58 & 7 & $3-38$ & 0.279 & $0.911 \pm 0.291$ & $0.270 \pm 0.624$ & $0.870 \pm 0.318$ \\
\hline Pe 84 & 4 & 338 & 14 & $10-43$ & 0.213 & $0.813 \pm 0.095$ & $0.005 \pm 0.039$ & $0.812 \pm 0.097$ \\
\hline Pe 99 & 6 & 39 & 7 & $3-19$ & 0.530 & $0.868 \pm 0.179$ & $-0.002 \pm 0.196$ & $0.867 \pm 0.166$ \\
\hline Pe103 & 4 & 40 & 7 & $3-20$ & 0.283 & $0.856 \pm 0.228$ & $0.003 \pm 0.193$ & $0.851 \pm 0.203$ \\
\hline All loci & & & 14 & & 0.430 & 0.849 & 0.010 & 0.848 \\
\hline $\begin{array}{l}\text { Jackknifing } \\
\text { over loci }\end{array}$ & & & & & & $0.850 \pm 0.014$ & $0.008 \pm 0.036$ & $0.848 \pm 0.014$ \\
\hline \multicolumn{9}{|c|}{ P. hoffmeyeri sp. B } \\
\hline Pe 84 & 3 & 115 & 12 & $1-14$ & 0.454 & $0.855 \pm 0.125$ & $0.009 \pm 0.132$ & $0.853 \pm 0.128$ \\
\hline Pe 99 & 2 & 60 & 10 & $1-11$ & 0.037 & 1.000 & 0.001 & 1.000 \\
\hline All loci* & & & 12 & & 0.245 & 0.879 & 0.016 & 0.877 \\
\hline \multicolumn{9}{|c|}{ P. gemellus sp. A } \\
\hline Pe 52 & 4 & 28 & 3 & $1-14$ & 0.432 & 0.690 & -0.066 & 0.709 \\
\hline Pe 77 & 4 & 28 & 3 & $1-14$ & 0.222 & 0.223 & -0.010 & 0.231 \\
\hline Pe 99 & 3 & 28 & 3 & $1-14$ & 0.274 & -0.012 & -0.051 & 0.037 \\
\hline All loci* & & & 3 & & 0.310 & 0.376 & -0.048 & 0.404 \\
\hline \multicolumn{9}{|c|}{ P. gemellus sp. B } \\
\hline Pe 28 & 16 & 228 & 9 & $13-48$ & 0.697 & $0.166 \pm 0.138$ & $0.001 \pm 0.018$ & $0.165 \pm 0.138$ \\
\hline Pe 52 & 19 & 229 & 9 & $13-48$ & 0.836 & $0.123 \pm 0.088$ & $-0.005 \pm 0.009$ & $0.127 \pm 0.090$ \\
\hline Pe 84 & 26 & 191 & 9 & $11-40$ & 0.892 & $0.212 \pm 0.113$ & $-0.001 \pm 0.012$ & $0.212 \pm 0.111$ \\
\hline Pe 99 & 5 & 227 & 9 & $12-48$ & 0.240 & $0.159 \pm 0.115$ & $-0.000 \pm 0.009$ & $0.159 \pm 0.118$ \\
\hline All loci & & & 9 & & 0.666 & 0.168 & -0.002 & 0.169 \\
\hline
\end{tabular}

Jackknifing over loci was not performed for less than five loci, for Pe 99 in P. hoffmeyeri sp. B, it was not possible to jackknife across crops because only the wasps from one crop were polymorphic.

to their detailed morphology, but to the fact that half of the species had not been noticed earlier in spite of extensive ecological, evolutionary, and taxonomic (Wiebes 1995) work on the system. Subsequent ongoing studies of their morphology shows that some cryptic species pairs show minor morphological differences (e.g. P. gemellus spp. $A$ and $B$ ), while others are more readily differentiated (e.g. P. hoffmeyeri spp. A and B) (R.D. Harrison, J.-Y. Rasplus, personal communication; personal observtion).

\section{Microsatellite protocols}

Two sets of samples were genotyped with microsatellites (Molbo et al. 2002). First, sampling a single female per fruit we collected 435 wasps from 14 crops of F. obtusifolia and 255 wasps from nine crops of $F$. popenoei. Crop is the term for fruit that develop simultaneously on an individual tree. These females were genotyped with two to five polymorphic loci to perform population genetic analyses (see Table 1 for sample sizes of individual loci and species). Different sets of loci were used for different species because amplification and variability of loci change from species to species. All loci that were variable and readable for each species were used.
Second, from F. obtusifolia fruits containing either more than one foundresses (46 fruits) or hybrid daughters (4 fruits), we genotyped larger samples of both male and female offspring with two loci (Pe 9 and Pe 84) to assign offspring to parents (see below for details).

For both samples, total genomic DNA was extracted from single individual wasps with the puregene ${ }^{\circledR}$ kit from Gentra using the manufacturer's protocol for single Drosophila with slight modifications (Molbo et al. 2002). DNA was resuspended in $50 \mu \mathrm{L}$ DNA rehydration buffer and for microsatellite analyses, $1 \mu \mathrm{L}$ of this was used in each polymerase chain reaction (PCR). PCR protocols are described in Molbo et al. (2002). Radiolabelled PCR products were separated by $6 \%$ polyacrylamide gel electrophoresis and visualized on X-ray film. Each gel was loaded with a known sequence and an individual of known genotype (or the sequenced clone) to facilitate scoring.

\section{Genetic structure within species}

We first tested for genotypic disequilibrium in all four pollinator species using the program FSTAT version 2.9.3 (Goudet 1995). Genotypic disequilibrium occurs when alleles 
at different loci appear not to be inherited independently. This can happen between neutral loci either when there is linkage (close physical proximity of loci on chromosomes) or when inbreeding or population structure prevents recombination between alleles at different loci.

Then, Weir \& Cockerham's (1984) estimates of genetic differentiation between crops $\left(F_{\mathrm{ST}}\right)$ and inbreeding $\left(F_{\mathrm{IS}}\right)$ were calculated using FSTAT (Goudet 1995) from the genotypes of $339^{\prime}$ ' $P$. hoffmeyeri sp. $A$ ' individuals, 115 'P. hoffmeyeri sp. $B^{\prime}$ individuals, $28^{\prime} P$. gemellus sp. $A$ ' individuals and 228 ' $P$. gemellus sp. $B$ ' individuals. The different sample sizes reflect the frequencies of the species in our collection. We calculated confidence intervals for the estimates of $F_{\mathrm{IT}}, F_{\mathrm{ST}}$ and $F_{\mathrm{IS}}$ by jackknifing over crops and over loci whenever more than four loci were available or wasps from more than four crops were analysed.

$F_{\mathrm{ST}}$ measures how much of the genetic variance is caused by the grouping variable. We used crops as the grouping variable in these analyses in order to detect either temporal, spatial or host-genotype related genetic differentiation between the pollinator samples. This means, that samples collected over different years were compared, including two cases in which three crops from the same individual F. obtusifolia tree were analysed as independent groups. We ran analyses of the entire dataset both pooling the crops from individual trees and keeping them as separate samples, and thereby determined that all crops could be used as independent groups (Compare the $95 \%$ confidence intervals from the 'independent crops' analysis presented in Table 1 with the values from jackknifing over all loci from the 'crops pooled within tree' analysis, where $P$. hoffmeyeri sp. $A$ has the values $F_{\mathrm{IT}}:(0.836-0.862) F_{\mathrm{ST}}:(-0.010-0.053)$ $F_{\mathrm{IS}}:(0.833-0.857)$ and for $P$. hoffmeyeri sp. $B$ the values are $\left.F_{\mathrm{IT}}: 0.877 F_{\mathrm{ST}}:-0.049 F_{\mathrm{IS}}: 0.883\right)$. Only the analysis where all crops are considered independent is presented in detail.

\section{Brood analyses and assignment to parents}

In 46 F. obtusifolia fruits where multiple foundresses were found we quantified the contributions of each foundress. The average number of dead foundresses in these fruits was $2.57 \pm 0.89 \mathrm{SD}$ and the harmonic mean number was 2.35. For each fruit we genotyped all males (or a maximum of 50) at the loci Pe 9 and Pe 84 and 10 females at Pe 9. The average number of males genotyped per fruit was $25 \pm 14$ SD. Genotypes of males and females were compared to reconstruct both parental genotypes. Offspring from a given foundress are referred to as belonging to the same matriline.

Four kinds of errors can occur when assigning offspring to matrilines. First, a matriline can remain undetected because no individuals of that matriline were collected. The risk of this error is very small because sex ratios are adjusted so that foundresses contributing few offspring to a shared brood produce a high proportion of males (Werren 1980; Kinoshita et al. 2002; Molbo et al. unpublished). By analysing all males in 38 broods and 50 males in the last eight, we virtually eliminated the risk of not sampling an existing matriline.

Second, when the only difference between two classes of offspring is their paternal alleles, it is not possible to determine whether they are the offspring of a doublemated mother or of two single mated mothers that have the same genotype. For brood analyses, we assumed that foundresses had mated only once. This assumption has not been tested, but in all but one of the analysed broods, the offspring that had different paternal alleles also differed in maternal alleles, indicating that multiple mating with genetically dissimilar males is very infrequent in these species.

Third, when heterozygous and homozygous females in a brood have the same paternal allele but two different maternal alleles, they could either be the offspring of a single heterozygous mother or two different mothers. In this case, we can infer the presence of several mothers when there are non-Mendelian proportions of the two offspring types. However, when genotypes occur in Mendelian proportions we cannot prove that there was only one mother. Only four of the observed matrilines consisted of a mixture of homo- and heterozygous females with an allele in common at one locus. Grouping males and females by their maternal allele gave the following genotype counts per Fig. 33:36, 12:14, 6:7 and 19:21, none of which exceeded one binomial standard error from the Mendelian proportions of equality. These four cases were each considered a single matriline.

The fourth error occurs when two foundresses as well as their mates have the same genotypes. In this case, individuals from different matrilines are indistinguishable. We estimated this risk by calculating the expected frequency of all possible genotype compositions of broods from any single-mated female (matriline types). The sum of all the squared matriline type frequencies gives the probability that two randomly drawn foundresses produce genetically indistinguishable broods. The expected genotype frequency in females was calculated from allele frequencies. The frequency of a homozygote AA is $P_{\mathrm{AA}}=F_{\mathrm{IS}} P_{\mathrm{A}}+P_{\mathrm{A}}^{2}\left(1-F_{\mathrm{IS}}\right)$, where $F_{\text {IS }}$ is the inbreeding coefficient for the locus in question (see Results, Table 1) and $P_{\mathrm{A}}$ is the frequency of allele A. The frequency of $\mathrm{AB}$ heterozygotes is $P_{\mathrm{AB}}=2 P_{\mathrm{A}} P_{\mathrm{B}}\left(1-F_{\mathrm{IS}}\right)$ and so forth for all possible genotypes. To calculate the frequencies of a given mating pair, the frequency of each female genotype was combined with the male genotype frequencies in a similar way. Because males are haploid, their genotype frequencies are identical to the population allele frequencies. Thus, the frequency of matings between AA females and A males $\left(P_{\mathrm{AA} \times \mathrm{A}}\right)$ is $P_{\mathrm{AA}} F_{\mathrm{IS}}+P_{\mathrm{AA}} P_{\mathrm{A}}\left(1-F_{\mathrm{IS}}\right)$. Similarly, the frequency of heterozygote females sharing 
an allele with her mate $\left(P_{\mathrm{AB} \times \mathrm{A}}\right)$ is $\left(P_{\mathrm{AB}} F_{\mathrm{IS}}\right) / 2+P_{\mathrm{AB}} P_{\mathrm{A}}\left(1-F_{\mathrm{IS}}\right)$. The first term is divided by two because half of the inbreeding $\mathrm{AB}$ females will mate with an A male, the other half with a $B$ male. Finally, the frequency of matings where no alleles are shared between partners $\left(P_{\mathrm{AB} \times \mathrm{C}}\right)$ is $P_{\mathrm{AB}} P_{\mathrm{C}}\left(1-F_{\mathrm{IS}}\right)$ because this can only occur in outbred matings. Overall, the probability of matrilines being genetically identical was 0.089 for 'P. hoffmeyeri sp. A' (locus Pe 9) and 0.375 for ' $P$. hoffmeyeri sp. $B$ ' (Pe 84). The higher value for 'P. hoffmeyeri sp. $B$ ' reflects the much lower genetic variation in this species. We used these estimated risks of nondetection to estimate the frequencies of two-matriline fruits from the observed numbers.

\section{Random association of species}

To determine whether foundresses of the two species associated randomly in figs, we compared the observed frequency of two-species fruits with the expected values if species associate randomly. The expected frequency of two-species fruits was calculated from the relative frequencies of each species and the frequency of twofoundress fruits. First, we determined the frequency of each cryptic species $(p, q)$ from one daughter taken from each of 75 fruits containing a single dead foundress. Then, we estimated the frequency of fruits with two matrilines $\left(\mathrm{N}_{2}\right)$ from the frequency of fruits with the two species together $\left(S_{2}\right)$ and the frequencies of either species $\left(N_{2}=S_{2} / 2 p q\right)$, assuming random association of foundresses of the two species.

From these values we estimated the expected brood type frequencies under the assumption that foundresses of the two species associate randomly (two broods of ' $P$. hoffmeyeri sp. $A$ ' $=p^{2} N_{2}$; two broods of ' $P$. hoffmeyeri sp. $B^{\prime}=q^{2} N_{2}$; two species broods $=2 p q N_{2}$; a single brood of ' $P$. hoffmeyeri sp. $A^{\prime}=p\left(1-N_{2}\right)$ and a single brood of ' $P$. hoffmeyeri sp. $\left.B^{\prime}=q\left(1-N_{2}\right)\right)$. These expected frequencies of brood types were compared with a $\chi^{2}$ test to the observed brood type frequencies corrected for the nondetection risk.

\section{Inbreeding calculated from foundress number distributions}

We compared our estimates of inbreeding with the two previously used indirect estimates of inbreeding (Frank 1985; Herre 1985). The main difference between these previous estimates is that Frank (1985) assumed that two foundresses produce twice the brood size of a single foundress, whereas Herre (1985) assumed that any number of foundresses produce the same number of daughters as a single foundress. The different brood size assumptions weigh the outbreeding from multifoundress broods differently. The two inbreeding estimates were calculated as follows:
(1) $F_{\text {(Herre) }}=1 /(4 \tilde{n}-3)$, where $\tilde{n}$ is the harmonic mean foundress number (Herre 1985).

(2) $F_{\text {(Frank) }}=-(R-1) /(2 R-1)$, where

$R=\left(x-\sqrt{x^{2}-4 y z}\right) / y$

$x=\sum \lambda_{n}\left(6 n^{2}-4 n+1\right) / 4 n$,

$y=\sum \lambda_{n}(4 n-1)(n-1) / 4 n$,

$z=\sum \lambda_{n}(2 n-1) n / 4 n$ and $\lambda_{n}$ is the frequency of fruits with foundress number $n$ (see Frank 1985 for further details).

For these calculations, we used two estimates of foundress numbers. First, the observed numbers of dead foundress bodies in the figs we collected. Second, because the foundress number distribution in our study was significantly different from the values reported in earlier studies (data from Herre 1989; $\chi^{2}=16.6, \mathrm{DF}=4, P=0.0023$ for $F$. obtusifolia; and $\chi^{2}=42.0, \mathrm{DF}=6, P<0.0001$ for $F$. popenoei), we also estimated inbreeding from the foundress number distribution reported in Herre's study (1989) which comprised 29 crops of F. obtusifolia compared to four in the present study and 23 crops of F. popenoei compared to six in the present study.

\section{Hybridization between species}

Hybrids were found in four fruits (Molbo et al. 2002, 2003; see Results). To determine whether introgression takes place, and whether hybrid frequencies departed from expectations based on random mating between species, the frequencies of hybrids were compared to the entire dataset of nonhybrid genotypes (one female per fruit). This comprised 435 females from F. obtusifolia, analysed with minimum the two loci Pe 9 and Pe 84 and 255 females from F. popenoei analysed with minimum the two loci Pe 52 and Pe 99 (see Table 1 for sample sizes at other loci).

From each of the fruits where hybrids were detected, six males and six females were analysed with the loci Pe 9 and Pe 84 to determine the maternal and paternal species and genotype. This analysis revealed the presence of hybrid diploid males (see Results). To verify this finding, genotyping was repeated at one locus. To determine whether the presence of diploid males was significantly associated with hybridization we compared the frequency of diploid males in hybrid and nonhybrid broods. For this comparison, only broods containing heterozygote females were used (six 'P. hoffmeyeri sp. $A$ ' matrilines and one 'sp. $B^{\prime}$ matriline). In four of these broods, all females were heterozygous, whereas in the three others $50 \%$ of the females were heterozygous at one locus. The probability of detection of diploid males was therefore $100 \%$ in the first four broods (60 males genotyped) and 50\% in the three others (76 males). 


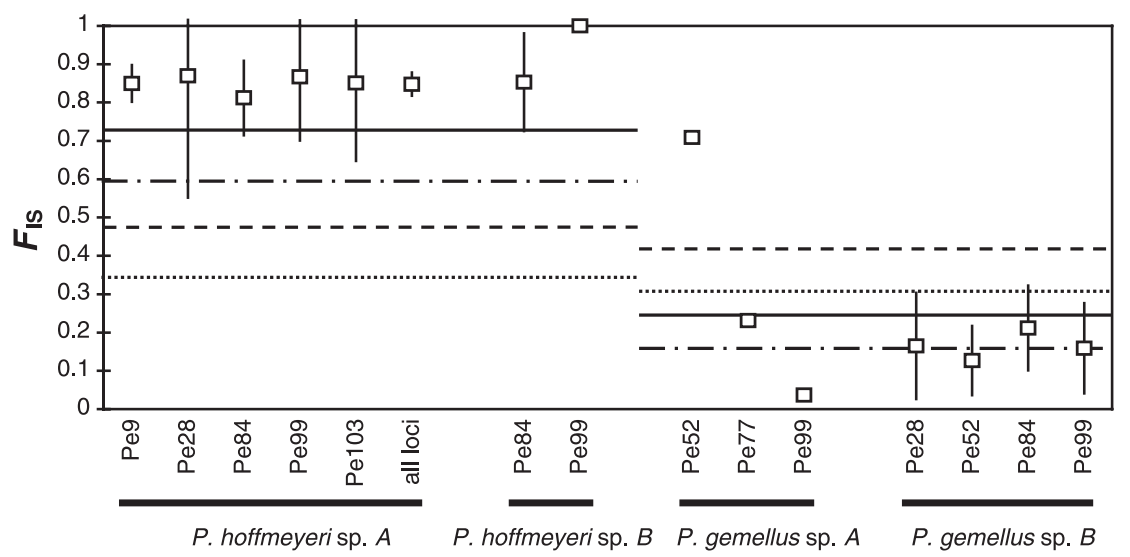

Fig. 1 Estimates of $F_{\text {IS }}$ with $95 \%$ confidence intervals from individual loci for each of the four pollinator species ' $P$. hoffmeyeri sp. $A$ ' and ' $P$. hoffmeyeri sp. $B$ ' associated with F. obtusifolia; 'P. gemellus sp. $A$ ' and 'P. gemellus sp. $B$ ' associated with $F$. popenoei and for all loci combined in 'P. hoffmeyeri sp. $A$ '. Samples that either did not have enough variation or too small sample size for jackknifing across crops, are presented without $95 \%$ confidence intervals. The four horizontal lines correspond to inbreeding estimates $F_{\text {Herre }}$ based on the foundress number distribution published in Herre (1989) (-), and from the present study (--), and $F_{\text {Frank }}$ based on the foundress number distribution published in Herre (1989) (---), and from the present study (‥). See methods for equations.

\section{Results}

\section{Population genetic analyses}

We found no significant genotypic disequilibrium between any pair of loci in any of the four species (all nominal $P$-values $>0.1$ ). There was also no evidence for genetic differentiation among crops within any of the four wasp species over the 4 years and c. $20 \mathrm{~km}$ separating samples. None of the $F_{\mathrm{ST}}$ values differed significantly from zero (Table 1). The different loci within a species all gave similar estimates of inbreeding $\left(F_{\mathrm{IS}}\right.$, Table 1). Important differences between loci were found only in 'Pegoscapus gemellus sp. $A$ ' with values ranging from 0.037 to 0.709 . However, this is the species with the smallest sample size (only 28 individuals), which probably accounts for the greater disagreement between loci. The inbreeding values were high for the four species and for none of the loci, where sample sizes allowed jackknifing, did the $95 \%$ confidence intervals from individual loci include zero (Table 1). In 'P. hoffmeyeri sp. $A^{\prime}$, data were available from enough loci to jackknife over loci, and the $F_{\text {IS }}$ value obtained that way was also significantly greater than zero. The two cryptic pollinator species from Ficus obtusifolia ('P. hoffmeyeri sp. A and B') were significantly more inbred than the two pollinators of F. popenoei ('P. gemellus $A$ and $B$ ') (Table 1, Fig. 1).

\section{Inbreeding estimates based on numbers of foundresses}

The inbreeding estimates calculated from the numbers of dead foundresses in the 121 fruits collected from $F$. obtusifolia were $F_{\text {Herre }}=0.472$ and $F_{\text {Frank }}=0.339$. These estimates were about half of, and differed significantly from the $F_{\mathrm{IS}}$ value directly measured from the genetic data (Fig. 1).
Using the foundress number distribution in the larger data set published by Herre (1989) yielded somewhat higher estimates of inbreeding but the values were still significantly lower than the $F_{\mathrm{IS}}$ value obtained from the genetic data (Fig. 1).

The difference between genetic and indirect foundress number-based measures of inbreeding was less pronounced for 'P. gemellus sp. $A$ and $B$ ' (Fig. 1). For ' $P$. gemellus sp. $A$ ' there was considerable difference in the estimate of $F_{\text {IS }}$ across loci and both the $F_{\text {(Herre) }}$ and $F_{\text {(Frank) }}$ estimates based on foundress number distributions published by Herre (1989) fell within the estimates from the three microsatellite loci. For ' $P$. gemellus sp. $B$ ', the $F_{(\text {Herre) }}$ estimate was higher than the estimate obtained for the four loci (and significantly so for one locus), whereas the $F_{\text {(Frank) }}$ estimate was within the $95 \%$ confidence intervals of all four loci (because data were obtained for only four loci, it was not possible to jackknife over loci).

\section{Multi-foundress brood composition}

Only 10 of the 46 F. obtusifolia fruits with two or more dead foundresses actually contained more than one distinct genetic matriline (Molbo et al. 2003). In five of these 10 fruits, the foundresses were from different species (Molbo et al. 2003).

Correcting for the risk of nondetection of two-foundress broods (0.089 in 'P. hoffmeyeri sp. $A$ ' and 0.375 in 'P. hoffmeyeri sp. $B^{\prime}$ see material and methods) had little effect on the estimated number of figs containing brood from more than one foundress (10.6 instead of 10.0). Table 2 gives the proportion of each type of association corrected for the probability of nondetection. These values were similar and not significantly different $(G=2.2925, \mathrm{DF}=1, P<0.1)$ from the expected values under the assumption that the two species associate randomly in figs (Table 2, see material and methods). 
Table 2 Observed and expected brood types among 121 fruits of F. obtusifolia, from which offspring was genotyped. Observed brood types are corrected for nondetection (see Materials and Methods)

\begin{tabular}{lll}
\hline Matrilines in the fruit & $\begin{array}{l}\text { Observed } \\
\text { corrected }\end{array}$ & Expected \\
\hline One 'P. hoffmeyeri sp. $A$ ' & 85.5 & 78.2 \\
One 'P. hoffmeyeri sp. $B^{\prime}$ & 25 & 30.4 \\
Two 'P. hoffmeyeri sp. $A$ ' & 5.6 & 6.4 \\
Two 'P. hoffmeyeri sp. $B$ ' & 0 & 1 \\
One 'P. hoffmeyeri sp. $A$ ' and one 'sp. B' & 5 & 5 \\
\hline
\end{tabular}

\section{Hybridization}

Hybrids were observed only between the two $P$. hoffmeyeri species (Molbo et al. 2002, 2003). Four of the 435 females (one per fruit) collected on F. obtusifolia were hybrids but none of the 255 females collected from $F$. popenoei were hybrids.

Assuming random mating and equal contribution to male and female production by the cofoundresses, the expected frequency of $P$. hoffmeyeri hybrids is half the frequency of figs containing both species, that is 0.041 . This value is not significantly different from the observed frequency of hybrids (0.009; Fisher's exact test, $P=0.175$, $\mathrm{DF}=1$ ), suggesting that individuals of the two cryptic species do not avoid allospecific matings when emerging in the same fig. The four observed hybrids were first generation hybrids and there was no evidence of introgression between species. All but those four individuals had genotypes that were fully consistent with being either pure ' $P$. hoffmeyeri sp. $A^{\prime}$ or 'sp. $B^{\prime}$ individuals.

From the four fruits where hybrid females were found, a total of 18 males were genotyped and assigned to the hybrid-producing matrilines. Two of these males (each from a different fruit) were diploid (they had the same hybrid genotypes as their diploid sisters). By contrast, not a single diploid male was found in the seven figs containing heterozygote nonhybrid females. Although the proportion of broods containing diploid males was not significantly greater among broods containing hybrid females (2/4) than among broods with nonhybrid females ( $0 / 7$; Fisher's exact test, $P=0.1091, \mathrm{DF}=1$ ), the proportion of diploid male individuals was significantly greater among males in broods with hybrid females $(2 / 18)$ than in those with nonhybrid females (0/98; Fisher's exact test, $P=0.0229, \mathrm{DF}=1)$.

The lack of hybrids between the $P$. gemellus species suggest that these two species do not mate with each other or fail to produce viable offspring. An estimated 18 cases of hybrids would have been expected among the 255 females genotyped, based on the species frequencies and dead foundress number distribution. The estimate assumes that the two species associate and mate randomly within fruits and all observed dead foundresses produced equal brood sizes. This significantly contrasts with our finding that none of the 255 females were hybrids (Fisher's exact $P$ value $<0.0001, \mathrm{DF}=1$ ).

\section{Discussion}

\section{Inbreeding}

The inbreeding levels were consistently high in these four fig pollinating wasp species, with the species characterized by lower foundress numbers showing higher levels of inbreeding. Both species associated with Ficus obtusifolia ('P. hoffmeyeri spp.') had higher $F_{\mathrm{IS}}$ values (and lower foundress numbers) than the two species associated with Ficus popenoei ('P. gemellus spp.'). A similar trend exists within the pairs of species that share hosts. The less common species of a pair has lower average foundress numbers and also higher inbreeding. However, jackknifing was not performed because of the small sample sizes and differences were not significant. The high $F_{\text {IS }}$ estimates reflect the breeding structure of fig wasps, and similar values are almost exclusively found in partially selfing organisms (Williams \& Guries 1994; Viard et al. 1997; Trouvé et al. 2003) or other organisms with extremely limited dispersal or local mating (Chesser 1983; Antolin 1999; Nevo et al. 2000; Murren 2003). The rarest of the four species, 'P. gemellus sp. $A^{\prime}$, was the only species where different loci gave very different $F_{\text {IS }}$ estimates. The small sample size available for this rare species probably accounts for its larger variation in $F_{\text {IS }}$ estimates.

Importantly, there were large and significant differences between the inbreeding values obtained by the genetic data and those predicted from the number of dead foundresses per fig. For the two pollinators of $F$. obtusifolia (' $P$. hoffmeyeri sp. $A$ ' and 'sp. $B$ ') the $F_{\text {IS }}$ values were about twice as high as the expected values based on the number of dead foundresses. This difference remained large and significant when using the distribution of foundress number from a much larger data set (recalculated from Herre 1989).

Our analyses of the brood genotypes revealed several reasons why the number of dead foundresses in F. obtusifolia yielded very biased estimates of inbreeding. First, 77\% of the fruits that contained multiple dead foundresses only had offspring from a single matriline. Thus, using the number of dead foundresses overestimates the opportunity for outbreeding. Second, a significant percentage of figs (nearly $50 \%$ ) with multiple matrilines actually contained different species. Since our data indicate that crosses between ' $P$. hoffmeyeri sp. $A$ ' and 'sp. $B$ ' do not produce fertile offspring, the number of foundresses overestimates the actual opportunity for outbreeding in such fruits. Finally, when several foundresses reproduced in a fig, there was frequently a significant skew in reproduction (Molbo et al. unpublished), 
Table 3 Change in mean brood size between fruits with one and two foundresses of either host fig species

\begin{tabular}{llllllll}
\hline $\begin{array}{l}\text { Foundress } \\
\text { number }\end{array}$ & \multicolumn{1}{l}{$N$} & $\begin{array}{l}\text { Mean } \\
\text { brood size }\end{array}$ & Std. dev. & DF & $t$-value & $P$-value \\
\hline F. obtusifolia & 1 & 75 & 224 & 55.3 & 103 & 0.131 & 0.8956 \\
& 2 & 30 & 222 & 67.3 & & & \\
F. popenoei & 1 & 95 & 111 & 40.4 & 150 & -9.40 & $<0.0001$ \\
& 2 & 57 & 199 & 75.2 & & & \\
\hline
\end{tabular}

which translates into a reduction in the effective number of matrilines per fruit. Depending on the precision of individual sex ratio adjustments, this may further increase the actual level of inbreeding (see also Molbo \& Parker 1996; Kathuria et al. 1999; Kinoshita et al. 2002).

In F. popenoei, the difference between inbreeding estimates based on the numbers of dead foundresses and those based on microsatellites was much lower for ' $P$. gemellus sp. $A$ ' and 'sp. $B$ '. This is potentially explained by two observations. First, ' $P$. gemellus sp. $A$ ' is very rare, and thus only a low proportion of the fruits with multiple foundresses hold two different species. Second, there is a better correspondence between the number of dead foundresses and the reproducing foundresses. Although we do not have genetic brood analyses from fruits of $F$. popenoei, we have indirect evidence from the increase in mean brood size from one- to two-foundress fruits (Table 3, Herre 1989). The mean number of offspring in fruits of $F$. popenoei with two dead foundresses is almost twice the number in fruits with only a single dead foundress. In contrast, the broods from two-foundress fruits of F. obtusifolia are not larger on average than broods from single foundress fruits (Table 3). This is in agreement with the low probability of second foundresses reproducing that we observed from brood genotypes.

In a recent study, Greeff (2002) used a body colour polymorphism to estimate the inbreeding level $\left(F_{\mathrm{IT}}\right)$ in another fig wasp species, Alfonsiella sp. In contrast to our study, he found that the actual level of inbreeding was lower than the value expected from foundress number distribution (observed: 0.683 ; expected: $0.723-0.850$ ). This discrepancy is probably due to the occurrence of a significant amount of male dispersal and mating outside the natal patch in Alfonsiella sp. Greeff's study further demonstrates the importance of using direct estimates of inbreeding when testing sex ratio theory.

\section{Population structure}

The genetic analyses revealed no significant genetic differentiation within any of the four wasp species. None of the $F_{\mathrm{ST}}$ values measured between crops were significantly greater than zero. These findings are in agreement with the biology of pollinating fig wasps. The synchrony of fruiting within a crop (Windsor et al. 1989) and the short adult lifespan of wasps (Kjellberg et al. 1988; Compton 1993) forces females to change host individual each generation. Crops have been shown to be pollinated by more than 10.7 (F. popenoei) and 17.3 (F. obtusifolia) different pollen donors (Nason et al. 1998). Moreover, female wasps routinely fly between 5.8 and $14.2 \mathrm{~km}$ (Nason et al. 1998), which is about the scale of the populations collected in this study. No trees sampled in this study were more than $20 \mathrm{~km}$ apart. Because our crops were collected over several years, the lack of significant genetic differentiation between crops also suggests a lack of genetic differentiation between temporally separated 'cohorts' of wasps. The adult, dispersing and mating wasp is thought to live only about two days out of a total lifespan of $c$. 6 weeks. Therefore, wasp 'cohorts' born more than a week apart will not overlap as adults and are unable to enter the same figs to lay their eggs. Such cohorts would be genetically separated, possibly for many generations. This separation however, appears not to be great enough to cause any genetic differentiation between cohorts. This indicates that in spite of the high inbreeding and population subdivision, the mating system of fig pollinating wasps does not in itself facilitate genetic population differentiation neither in time nor at geographical scales of 10-20 kilometres.

The pattern of high levels of inbreeding combined with $F_{\mathrm{ST}}$ values of zero reflects the mating and dispersal patterns of fig wasps. The high level of sibmating combined with long distance migration each generation, and a low frequency of random outbreeding gives a very different population genetic pattern from most other inbred animal populations. In most animals, limited dispersal distances cause high levels of inbreeding closely coupled with high levels of population differentiation. In contrast, the population genetics of LMC species resemble those of partially selfing organisms (see Williams \& Guries 1994; Viard et al. 1997; Trouvé et al. 2003).

\section{Hybridization}

We found no evidence of genetic introgression between species, although the two 'P. hoffmeyeri spp.' were hybridizing. All the hybrids observed were F1 hybrids, suggesting that hybrids have severely reduced fitness if any at all. Interestingly, our data suggested that the frequency of hybrid matrilines was not significantly lower than half the frequency of fruits with two species. Although the sample size was relatively small, this suggests that the two species do not avoid interspecific matings. A possible explanation for this is that the low frequency of two-species broods causes only weak selection against interspecific matings. 
Among the two 'P. gemellus spp.', no hybrids were observed. This lack of hybrids could be due to two factors that distinguish these wasps from the hybridizing $P$. hoffmeyeri species. First, the two P. gemellus species are considerably older and more diverged (Molbo et al. 2003; Mean COI divergence between 'P. gemellus spp.' is 6.62 compared to 4.43 between ' $P$. hoffmeyeri spp.'). Second, the average foundress numbers per fruit are much higher for ' $P$. gemellus spp.', which means the rarer species will almost always share fruit with the more common species, and thus be under much stronger selection to avoid unfertile allospecific matings.

Two of the four figs that contained $P$. hoffmeyeri species hybrid females contained diploid males. In each of these broods, a single out of six males was diploid. No diploid males were found among the offspring produced by conspecifically mated females. This suggests that hybridization causes a breakdown of the sex determination system since normal hymenopteran males develop from unfertilized eggs and are haploid (Cook 1993; Dobson \& Tanouye 1998). A dysfunctional sex determination system could be one of the proximate mechanisms involved in the reduction of hybrid fitness. Diploid males are often sterile in Hymenoptera (Cook 1993), and other vital genes may be dysfunctional in hybrid females.

\section{Local mate competition theory}

The findings of this study have important implications for earlier tests of Hamilton's local mate competition (LMC) theory using fig wasps (Hamilton 1979; Frank 1985; Herre 1985; Herre 1987; Herre et al. 1997). First, and most importantly, although previous studies found consistent qualitative agreement between data and predictions that were based on several explicit assumptions, our study shows that the numbers of dead foundresses do not accurately estimate the breeding structure and the level of inbreeding. The observed higher inbreeding levels in F. obtusifolia pollinators means that the optimal sex ratio at least in this case is more female biased than previously estimated. Second, researchers cannot necessarily assume that the number of bodies of potential foundresses found within a fig fruit provides an accurate guide to the number of foundresses that actually contributed to the broods (and brood sex ratios) found within (see also Kathuria et al. 1999; Kinoshita et al. 1998, 2002). Indeed, we often found that in figs that contained the bodies of several potential foundresses, only one of them actually reproduced. This indicates the importance of correctly assigning foundress number to brood composition in order to correctly test predictions of theory. In this context it will be important to determine whether the sex ratios in broods with several potential foundresses but with a single female monopolizing reproduction differ from broods in which only one foundress body was found. This will give information on the cues used by foundresses to adjust the sex ratio of their brood. Third, our study shows that the reasons for the generally female biased discrepancy between data and theory observed in earlier studies could be much simpler than some of the possibilities that have been suggested by their authors. For example, two previous studies have attempted to reconcile the discrepancies between theoretical predictions and observed sex ratios by altering some of the implicit parameters involved in generating the predictions. First, Hamilton (1979) posited a relative disadvantage of 0.78 to a chromosome from lack of recombination, which would give outbred females a higher fitness relative to inbred females and haploid males. This hypothesized inbreeding depression produced a much better fit between the modified theory and the data that he collected (Hamilton 1979). Second, Frank (1985) suggested that population differentiation either in time or in space between groups of foundresses settling on receptive figs (corresponding to our use of crop as grouping variable), would result in a type of hierarchical group selection that could also select for more female biased brood sex ratios. He suggested a panmictic index $P_{\mathrm{ST}}$ of 0.671 (where $P_{\mathrm{ST}}=1-F_{\mathrm{ST}}$ ) would reconcile his observed sex ratios with predictions.

Our data do not support the high inbreeding depression that Hamilton posited, and our direct estimates of $F_{\mathrm{ST}}$ suggest that higher order hierarchical structure is unlikely to play any significant role in sex ratio evolution in these fig wasps (see also Nason et al. 1998; Herre et al. 2001). Instead, our data suggest that the most likely explanations for the observed 'too female biased sex ratios' are a lack of direct relationship between foundress numbers and breeding structure in some species of fig wasps.

\section{Acknowledgements}

We wish to thank A. Gomez, M. Lopez and K. Refslund Petersen for help in the field, M.J.B. Krieger, C. Roger and K. Parker for advice and help in the laboratory. We thank A.N.M. Bot, S.A. West, S.J. Meier, R.D. Harrison and two anonymous reviewers for commenting on previous versions of the manuscript. We also wish to thank the Swiss National Science foundation for several grants to LK, Smithsonian Tropical Research Institute for a shortterm fellowship for DM, Augustinus fondet (Denmark) and the Swiss federal grants for foreign students for funding DM during parts of this project.

\section{References}

Antolin MF (1999) A genetic perspective on mating systems and sex ratios of parasitoid wasps. Researches on Population Ecology, 41, 29-37.

Boomsma JJ, Grafen A (1991) Colony-level sex ratio selection in the eusocial Hymenoptera. Journal of Evolutionary Biology, 3, 383-407.

Charnov EL (1982) The Theory of Sex Allocation. Monographs in population biology. Princeton University Press, Princeton, New Jersey. 
Chesser RK (1983) Genetic variability within and among populations of the black-tailed prairie dog. Evolution, 37, 320-331.

Compton SG (1993) One way to be a fig. African Entomolgy, 1, 151158.

Cook JM (1993) Sex determination in the Hymenoptera: a review of models and evidence. Heredity, 71, 421-435.

Dobson SL, Tanouye MA (1998) Evidence for a genomic imprinting sex determination mechanism in Nasonia vitripennis (Hymenoptera; Chalcidoidea). Genetics, 149, 233-242.

Fisher RA (1958) The Genetical Theory of Natural Selection. Dover publications, New York.

Frank SA (1984) The behavior and morphology of the fig wasps Pegoscapus assuteus and P. jimenezi: descriptions and suggested behavioral characters for phylogenetic studies. Psyche, 91, 289-308.

Frank SA (1985) Hierarchical Selection Theory and Sex Ratios. II. On Applying the Theory, and a Test with Fig Wasps. Evolution, 39, 949-964.

Goudet J (1995) FSTAT (version 1.2): a computer program to calculate F-statistics. Journal of Heredity, 86, 485-486.

Greeff JM (1997) Offspring allocation in externally ovipositing fig wasps with varying clutch size and sex ratio. Behavioral Ecology, 8, 500-505.

Greeff JM (2002) Mating system and sex ratios of a pollinating fig wasp with dispersing males. Proceedings of the Royal Society Biology Sciences Series B, 269, 2317-2323.

Greeff JM, Compton SG (1996) Sequential oviposition and optimal sex ratios in pollinating fig wasps. Ecological Entomology, 21, 300-302.

Hamilton WD (1967) Extraordinary sex ratios; A sex ratio theory for sex linkage and inbreeding has new implications in cytogenetics and entomology. Science, 156, 477-488.

Hamilton WD (1979) Wingless and fighting males in fig wasps and other insects. In: Sexual Selection and Reproductive Competition in Insects (eds Blum MS, Blum NA), pp. 167-220. Academic Press, New York.

Hardy ICW, ed. (2002) Sex Ratios, Concepts and Research Methods. Cambridge University press, Cambridge.

Herre EA (1985) Sex Ratio Adjustment in Fig Wasps. Science, 228, 896-898.

Herre EA (1987) Optimality, plasticity and selective regime in fig wasp sex ratios. Nature, 329, 627-629.

Herre EA (1989) Coevolution of reproductive characteristics in 12 species of New World figs and their pollinator wasps. Experientia, 45, 637-647.

Herre EA, Machado CA, West SA (2001) Selective regime and fig wasp sex ratios: towards sorting rigor from pseudo-rigor in tests of adaptation. In: Adaptationism and Optimality (eds Orzack SH, Sober E), pp. 191-218. Cambridge University press, Cambridge.

Herre AE, West SA, Cook JM, Compton SG, Kjellberg F (1997) Fig Wasp mating systems: Pollinators and Parasites, Sex Ratio Adjustment and Male Polymorphism, Population Structure and its Consequences. In: Social Competition and Cooperation in Insects and Arachnids I. Evolution of Mating Systems (eds Choe JC, Crespi B), pp. 226-239. Cambridge University press, Cambridge.

Kathuria P, Greeff JM, Compton SG, Ganeshaiah KN (1999) What fig wasp sex ratios may or may not tell us about sex allocation strategies. Oikos, 87, 520-530.

Kinoshita M, Kasuya E, Yahara T (1998) More highly femalebiased sex ratio in the fig wasp, Blastophaga nipponica Grandi (Agaonidae). Researches on Population Ecology, 40, 239-242.
Kinoshita M, Kasuya E, Yahara T (2002) Effects of time-dependent competition for oviposition sites on clutch sizes and offspring sex ratios in a fig wasp. Oikos, 96, 31-35.

Kjellberg F, Doumesche B, Bronstein JL (1988) Longevity of a fig wasp (Blastophaga psenes). Proceedings of the Koninklijke Nederlandse Akademie Van Wetenschappen-Biology Chemical Geological Physical and Medical Sciences, 91, 117-122.

Komdeur J, Pen I (2002) Adaptive sex allocation in birds: the complexities of linking theory and practice. Philosophical transactions of the Royal Society of London - Series B: Biology Sciences, 357, 373380.

Molbo D, Krieger MJB, Herre EA, Keller L (2002) Species-diagnostic microsatellite loci for the fig wasp genus Pegoscapus. Molecular Ecology Notes, 2, 440-442.

Molbo D, Machado CA, Sevenster JG, Keller L, Herre EA (2003) Cryptic species of fig pollinating wasps: Implications for the evolution of the fig-wasp mutualism, sex allocation and precision of adaptation. Proceedings of the National Academy of Sciences of the United States of America, 100, 5867-5872.

Molbo D, Parker ED Jr. (1996) Mating structure and sex ratio variation in a natural population of Nasonia vitripennis. Proceedings of the Royal Society of London - Series B: Biology Sciences, 263, 1703-1709.

Murren CJ (2003) Spatial and demographic population genetic structure in Catasetum viridiflavum across a human-disturbed habitat. Journal of Evolutionary Biology, 16, 333-342.

Nason JD, Herre EA, Hamrick JL (1998) The Breeding Structure of a Tropical Keystone Plant Resource. Nature, 391, 685-687.

Nevo E, Beiles A, Korol AB, Ronin YI, Pavlicek T, Hamilton W (2000) Extraordinary multilocus genetic organization in mole crickets, gryllotalpidae. Evolution, 54, 586-605.

Seger J, Eckhart VM (1996) Evolution of sexual systems and sex allocation in plants when growth and reproduction overlap. Proceedings of the Royal Society of London - Series B: Biology Sciences, 263, 833-841.

Stubblefield JW, Seger J (1990) Local mate competition with variable fecundity: dependence of offspring sex ratios on information utilization and mode of male production. Behavioral Ecology, 1, 68-80.

Trivers RL, Hare H (1976) Haplodiploidy and the evolution of social insects. Science, 191, 249-263.

Trivers RL, Willard DE (1973) Natural selection of parental ability to vary the sex ratio of offspring. Science, 179, 90-92.

Trouvé S, Degen L, Renaud F, Goudet J (2003) Evolutionary implications of a high selfing rate in the freshwater snail Lymnaea truncatula. Evolution, 57, 2303-2314.

Viard F, Justy F, Jarne P (1997) The influence of self-fertilization and population dynamics on the genetic structure of subdivided populations: a case study using microsatellite markers in the freshwater snail Bulinus truncatus. Evolution, 51, 15181528.

Weir BS, Cockerham CC (1984) Estimating F-statistics for the analysis of population structure. Evolution, 38, 1358-1370.

Werren JH (1980) Sex Ratio Adaptations to Local Mate Competition in a Parasitic Wasp. Science, 208, 1157-1159.

Werren JH (1983) Sex ratio evolution under local mate competition in a parasitic wasp. Evolution, 37, 116-124.

West SA, Herre EA (1998) Stabilizing Selection and Variance In Fig Wasp Sex Ratios. Evolution, 52, 475-485.

West SA, Herre EA, Sheldon BC (2000) The benefits of allocating sex. Science, 290, 288-290. 
Wiebes JT (1995) Agaonidae (Hymenoptera, chalcidoidea) and Ficus (Moracea): Fig wasps and their figs, XV (Mesoamerican Pegoscapus). Proceedings of the Koninklijke Nederlandse Akademie Van Wetenschappen, 98, 167-183.

Williams CF, Guries RP (1994) Genetic consequences of seed dispersal in three sympatric forest herbs. I. Hierarchical populationgenetic structure. Evolution, 48, 791-805.

Windsor DM, Morrison DW, Estribi MA, de Leon B (1989) Phenology of fruit and leaf production by 'strangler' figs on Barro Colorado Island, Panamá. Experientia, 45, 647-653.
The four authors started working together as collaborators and advisors during Drude Molbo's PhD work which includes the work presented here. They united a great combination of molecular, ecological and theoretical expertise with a common interest in evolutionary questions (and fig wasps). Specifically, these interests range from sex ratio adaptation over host-symbiont coevolution (Wolbachia with fig wasps and fig wasps with Ficus), to speciation and molecular evolution. 